УДК 611.41-073.43-055.2-053.8 (477.62)

DOI: $10.26435 /$ UC.V0I3(36).483

\author{
А.И. Кулиш, О.А. Бешуля \\ ГОО ВПО «Донецкий национальный медицинский университет имени М. Горького», Донецк
}

\title{
КОЛИЧЕСТВЕННАЯ АНАТОМИЯ СЕЛЕЗЕНКИ ПО ДАННЫМ УЛЬТРАЗВУКОВОГО ИССЛЕДОВАНИЯ ЛИЦ ЖЕНСКОГО ПОЛА ДОНЕЦКОГО РЕГИОНА
}

\footnotetext{
Селезенка - это крупный периферический лимфоидный орган, выполняющий фильтрационную, очистительную, иммунную, кроветворную и депонирующую функции [1]. Структура селезенки изменяется в зависимости от функционального состояния организма, с чем связана малочисленность и противоречивость данных относительно ее строения [2]. Соответственно, размер селезенки говорит о ее функциональном состоянии, поэтому определение диапазона нормальных показателей органа у здорового человека стало актуальной задачей для ученых. Наиболее информативным, неинвазивным, безопасным и относительно недорогим методом оценки размеров селезенки является ультразвуковое исследование, позволяющее получить наиболее точные данные о размерах органа и его структуре. На данный момент нет единого мнения о том, какие размеры селезенки необходимо считать нормальными и ведется дискуссия об ультразвуковых критериях, характеризующих нормальное состояние селезенки как у детей, так и у взрослых [3]. Большинство авторов указывают, что два линейных размера селезенки достаточны для определения увеличения размера органа и для контроля размеров селезенки в динамике [4]. Длина селезенки в норме, по данным одних авторов, составляет 10-12 (до 14) см [5,6], по другим данным, длина не должна превышать 15 см [7], ширина 7-8 см [8] и толщина - 5-7 см $[9,10]$.

Отличительной чертой анатомии селезенки является значительная вариабельность ее размеров, строения связочного аппарата, архитектоники у лиц с разным типом телосложения [11, 12]. Конституция имеет огромное значения для уточнения типологических проявлений в диагностике, прогнозировании и терапии первичной и вторичной патологии селезенки, оценке факторов, которые влияют и или предупреждают ее развитие [13].
}

\section{ЦЕЛЬРАБОТЫ}

Определить количественные параметры селезенки по данным ультразвукового исследования (УЗИ) с учетом антропометрических параметров у лиц женского пола первого периода зрелого возраста Донецкого региона для установления возрастной нормы.

\section{МАТЕРИАЛ И МЕТОДЫ}

Исследование было одномоментным (поперечным) и осуществлялось на основе научной методологии доказательной медицины. У всех обследованных было получено информированное согласие на участие в исследовании.

Обязательным условием отбора участников было четкое соблюдение условий включения в исследование. Условия включения: лица женского пола, первого периода зрелого возраста - 21-35 лет; условно здоровые (по результатам ежегодного диспансерного наблюдения); отсутствие вредных привычек (курение, злоупотребление алкоголем и др.). Критериями исключения при этом служили анамнестические указания на заболевания или травмы селезенки, жалобы на состояние здоровья в момент исследования. Всего было обследовано 50 человек.

Антропометрическое исследование проводили по общепринятой методике [14] в утренние часы в специально оборудованном кабинете. При обследовании использовали следующий инструментарий: вертикальный антропометр Мартина с градуировкой до 1 мм и медицинские весы. Затем производили расчет индекса Кетле, иногда называемый индексом Кетле-ГульдаКаупа или просто индексом массы тела (ИМТ) по формуле [15]:

ИМТ $=($ масса тела, кг $) /(\text { длина тела, м })^{2}$.

() А.И. Кулиш, О.А. Бешуля, 2020

(c) Университетская Клиника, 2020 
Классификация значений ИМТ, предложенная ВОЗ

Таблица 1.

\begin{tabular}{cc}
\hline ИМТ, кг/м2 & Классификация \\
\hline Менее 18,5 & Дефицит массы тела; \\
\hline $18,5-24,9$ & Нормальная масса тела; \\
\hline $25,0-29,9$ & Избыточная масса тела \\
\hline $30,0-34,9$ & Ожирение І степени \\
\hline $35,0-39,9$ & Ожирение II степени \\
\hline Свыше 40 & Ожирение ІІІ степени \\
\hline
\end{tabular}

Оценивали по классификации значений ИМТ, предложенной ВОЗ [16] (табл. 1.).

Ультразвуковое исследование выполняли по определенной методике одним оператором в В-режиме серой шкалы в стандартных положениях (на спине и на боку), при продольном и поперечном сканировании аппаратом Radmir, используя конвексный датчик. Измеряли ряд количественных показателей: длину селезенки (расстояние между передним и задним концами), ширину селезенки (расстояние между верхним и нижним краями) и толщину селезенки (расстояние между наружной и внутренней поверхностями на уровне ворот). Объем селезенки рассчитывали по формуле:

$\mathrm{OC}=0,6 \times \mathrm{a} \times \mathrm{b} \times \mathrm{c}$,

где a - длина селезенки, b - ширина, с - толщина органа [17].

Полученные данные заносились в таблицы Excel, после чего были статистически обработаны в программе Statistica 10.0.

\section{РЕЗУЛЬТАТЫ И ОБСУЖДЕНИЕ}

На первом этапе работы были произведены антропометрические замеры (рост и вес) и на основании полученных данных рассчитан ИМТ. По классификации значений ИМТ, предложенной ВОЗ, установили и поделили исследуемый контингент на 3 группы: группа 1 - женщины с нормальной массой тела, что составило 74\%; группа 2 - с дефицитом массы тела 7 человек (14\%), 6 человек с избыточной массой тела (12\%) - группа 3. На втором этапе - проведено ультразвуковое исследование селезенки.

Далее, все полученные результаты проверены на соответствие нормальному закону распределения частот величин, для чего использовали критерий Шапиро-Уилка.

В 1-й группе установили, что все изучаемые показатели не отличается от нормального закона распределения на уровне значимости $\mathrm{p}=>0,05$ : для показателя длины селезенки уровень значимости составил $p=0,73$, ширины $p=0,61$, тол- щины $\mathrm{p}=0,76$, объема $\mathrm{p}=0,83$; роста $\mathrm{p}=0,64$, веса $\mathrm{p}=0,39$, ИМТ $=0,06$.

Во 2-й группе установили, что следующие показатели не отличается от нормального закона распределения на уровне значимости $\mathrm{p}=>0,05$ : для показателя длины селезенки уровень значимости составил $\mathrm{p}=0,65$, ширины $\mathrm{p}=0,33$, толщины $\mathrm{p}=0,27$, объема $\mathrm{p}=0,43$, роста $\mathrm{p}=0,5$, веса $\mathrm{p}=0,97$. Показатель ИМТ не подчиняется нормальному закону распределения частот величин на уровне значимости $\mathrm{p}=0,03$.

В 3-й группе установили, что следующие показатели не отличается от нормального закона распределения на уровне значимости $\mathrm{p}=>0,05$ : для показателя длины селезенки уровень значимости составил $\mathrm{p}=0,32$, ширины $\mathrm{p}=0,72$, объема $\mathrm{p}=0,95$, роста $\mathrm{p}=0,2$, веса $\mathrm{p}=0,39$, ИМТ $\mathrm{p}=0,7$. Показатель толщины не подчиняется нормальному закону распределения частот величин на уровне значимости $\mathrm{p}=0,02$.

Для выборки лиц с нормальной массой тела получены следующие результаты: по данным УЗИ среднее значение длины селезенки оказа-

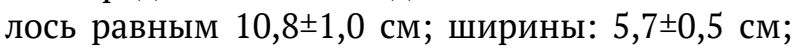

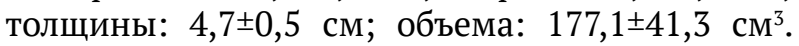
По данным антропометрии: среднее значе-

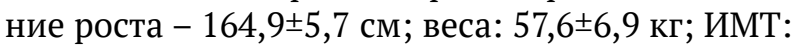
$21,1 \pm 1,8$.

В выборке лиц с дефицитом массы тела получены следующие результаты: по данным УЗИ среднее значение длины селезенки 10,6士0,8 см;

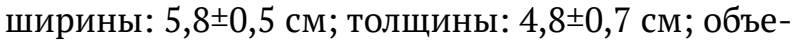
ма: $175,4 \pm 28,7$ см$^{3}$. Для показателей антропометрии среднее значение роста $-167,7 \pm 6,9$ см; веса:

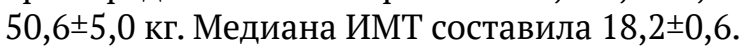

У выборки лиц с избыточной массой тела получены следующие результаты: в соответствии с данными УЗИ среднее значение длины органа

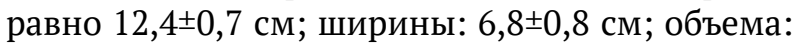
$258,5 \pm 57,9$ см3. Медиана толщины селезенки со-

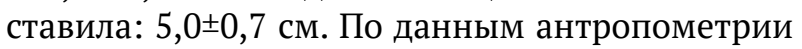
среднее значение роста - 167,8 $\pm 5,3$ см; веса:

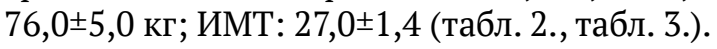


Нормальные размеры селезенки у взрослых имеют достаточно большую вариабильность, в связи с этим говорить о спленомегалии достаточно сложно. Увеличение селезенки диагностируют при длине селезенки более 12 см, толщину и ширину считают дополнительными измерениями, более 5 см и 7 см соответственно [9]. На
УЗИ норма размеров селезенки у взрослых считается относительно постоянным показателем, но может варьировать в зависимости от роста пациента и его массы тела. Так, люди с высоким ростом и избыточным весом могут иметь селезенку с «увеличенными» размерами, что важно учитывать врачу-диагносту при написании за-

Показатели описательной статистики количественных параметров селезенки

Таблица 2. у лиц женского пола первого периода зрелого возраста с распределением на группы по индексу массы тела

\begin{tabular}{|c|c|c|c|c|c|c|c|c|c|c|c|c|}
\hline \multirow{2}{*}{ Показатель } & \multicolumn{3}{|c|}{$\begin{array}{c}\text { Длина } \\
\text { селезенки }\end{array}$} & \multicolumn{3}{|c|}{$\begin{array}{c}\text { Ширина } \\
\text { селезенки }\end{array}$} & \multicolumn{3}{|c|}{$\begin{array}{c}\text { Толщина } \\
\text { селезенки }\end{array}$} & \multicolumn{3}{|c|}{$\begin{array}{c}\text { Объем } \\
\text { селезенки }\end{array}$} \\
\hline & 1 & 2 & 3 & 1 & 2 & 3 & 1 & 2 & 3 & 1 & 2 & 3 \\
\hline Число наблюдений & 37 & 7 & 6 & 37 & 7 & 6 & 37 & 7 & 6 & 37 & 7 & 6 \\
\hline $\begin{array}{l}\text { Среднее } \\
\text { значение, см }\end{array}$ & 10,8 & 10,6 & 12,4 & 5,7 & 5,8 & 6,8 & 4,7 & 4,8 & - & 177,1 & 175,4 & 258,5 \\
\hline Медиана & - & - & - & - & - & - & - & - & 5,0 & - & - & - \\
\hline $\begin{array}{l}\text { Доверительный } \\
\text { интервал -95\% }\end{array}$ & 10,5 & 9,9 & 11,7 & 5,6 & 5,3 & 6,0 & 4,5 & 4,1 & 4,3 & 163,3 & 148,9 & 197,8 \\
\hline $\begin{array}{l}\text { Доверительный } \\
\text { интервал +95\% }\end{array}$ & 11,1 & 11,4 & 13,2 & 5,9 & 6,2 & 7,5 & 4,9 & 5,4 & 5,9 & 190,8 & 202,0 & 319,2 \\
\hline $\begin{array}{l}\text { Минимальное } \\
\text { значение, см }\end{array}$ & 8,7 & 9,6 & 11,2 & 4,3 & 5,1 & 5,6 & 3,5 & 3,8 & 4,5 & 84,9 & 144,4 & 177,3 \\
\hline $\begin{array}{l}\text { Максимальное } \\
\text { значение, см }\end{array}$ & 13,0 & 11,7 & 13,1 & 7,0 & 6,4 & 7,7 & 5,8 & 6,1 & 6,5 & 278,3 & 228,6 & 345,5 \\
\hline Нижняя квартиль & 10,1 & 9,8 & 12,2 & 5,5 & 5,3 & 6,1 & 4,4 & 4,5 & 4,7 & 151,4 & 147,4 & 232,2 \\
\hline Верхняя квартиль & 11,5 & 11,3 & 13,0 & 6,1 & 6,2 & 7,2 & 5,1 & 4,9 & 5,0 & 199,6 & 187,4 & 298,0 \\
\hline $\begin{array}{l}\text { Стандартное } \\
\text { отклонение }\end{array}$ & 1,0 & 0,8 & 0,7 & 0,5 & 0,5 & 0,8 & 0,5 & 0,7 & 0,7 & 41,3 & 28,7 & 57,9 \\
\hline $\begin{array}{l}\text { Стандартная } \\
\text { ошибка }\end{array}$ & 0,2 & 0,3 & 0,3 & 0,1 & 0,2 & 0,3 & 0,1 & 0,3 & 0,3 & 6,8 & 10,9 & 23,6 \\
\hline
\end{tabular}

Показатели описательной статистики изучаемых параметров антропометрии

Таблица 3. у лиц женского пола первого периода зрелого возраста с распределением на группы по индексу массы тела

\begin{tabular}{lccccccccc}
\hline \multirow{2}{*}{\multicolumn{1}{c}{ Показатель }} & \multicolumn{3}{c}{ Рост } & \multicolumn{3}{c}{ Вес } & \multicolumn{3}{c}{ Индекс массы тела } \\
\cline { 2 - 10 } & 1 & 2 & 3 & 1 & 2 & 3 & 1 & 2 & 3 \\
\hline \hline Число наблюдений & 37 & 7 & 6 & 37 & 7 & 6 & 37 & 7 & 6 \\
\hline Среднее значение, см & 164,9 & 167,7 & 167,8 & 57,6 & 50,6 & 76,0 & 21,1 & - & 27,0 \\
\hline Медиана & - & - & - & - & - & - & - & 18,2 & - \\
\hline Доверительный интервал -95\% & 163,0 & 161,3 & 162,3 & 55,3 & 46,0 & 70,7 & 20,5 & 17,4 & 25,5 \\
\hline Доверительный интервал +95\% & 166,8 & 174,1 & 173,4 & 59,9 & 55,2 & 81,3 & 21,7 & 18,5 & 28,4 \\
\hline Минимальное значение, см & 154,0 & 160,0 & 158,0 & 45,0 & 43,0 & 67,0 & 18,6 & 16,8 & 25,4 \\
\hline Максимальное значение, см & 178,0 & 178,0 & 173,0 & 77,0 & 58,0 & 82,0 & 24,9 & 18,4 & 29,1 \\
\hline Нижняя квартиль & 161,0 & 160,0 & 167,0 & 54,0 & 46,0 & 75,0 & 19,5 & 17,6 & 25,7 \\
\hline Верхняя квартиль & 168,0 & 175,0 & 171,0 & 60,0 & 54,0 & 78,0 & 22,6 & 18,3 & 28,0 \\
\hline Стандартное отклонение & 5,7 & 6,9 & 5,3 & 6,9 & 5,0 & 5,0 & 1,8 & 0,6 & 1,4 \\
\hline Стандартная ошибка & 0,9 & 2,6 & 2,2 & 1,1 & 1,9 & 2,1 & 0,3 & 0,2 & 0,6 \\
\hline
\end{tabular}


ключения [13]. Потому, в спорных вопросах необходимо измерять не только длину селезенки, но и ее толщину и ширину, с учетом роста и веса обследуемого.

\section{В Ы В О Д Ы}

В результате исследования были определены количественные параметры селезенки с учетом антропометрических данных у лиц женского пола первого периода зрелого возраста жителей Донецкого региона.

\section{А.И. Кулиш, О.А. Бешуля \\ ГОО ВПО «Донецкий национальный медицинский университет имени М. Горького», Донецк \\ КОЛИЧЕСТВЕННАЯ АНАТОМИЯ СЕЛЕЗЕНКИ ПО ДАННЫМ УЛЬТРАЗВУКОВОГО ИССЛЕДОВАНИЯ ЛИЦ ЖЕНСКОГО ПОЛА ДОНЕЦКОГО РЕГИОНА}

В настоящее время вопросы индивидуальной изменчивости размеров селезенки и ее производных с учетом индекса массы тела у лиц женского пола 1-го периода зрелого возраста Донецкого региона практически не исследованы, что делает изучение данного вопроса актуальным.

Цель работы. Определить количественные параметры селезенки по данным ультразвукового метода исследования с учетом антропометрических параметров у лиц женского пола первого периода зрелого возраста Донецкого региона для установления возрастной нормы.

Материал и методы. Использовали опрос, антропометрическое исследование, ультразвуковое исследование селезенки, статистический анализ. Антропометрическое исследование проводили по общепринятой методике: измеряли рост и вес исследуемых. Ультразвуковое исследование выполняли по определенной методике одним оператором в В - режиме серой шкалы в стандартных положениях, при продольном и поперечном сканировании. Измеряли ряд количественных показателей: длину, ширину и толщину селезенки. Полученные данные заносились в таблицы Excel, после чего были статистически обработаны в программе Statistica 10.0.

Результаты и обсуждение. Для выборки лиц с нормальной массой тела получены следующие результаты: по данным ультразвукового исследования среднее значение длины селезенки оказалось равным

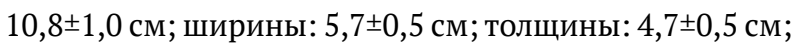

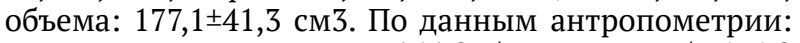
среднее значение роста - $164,9 \pm 5,7$ см; веса: $57,6 \pm 6,9$ кг; индекса массы тела: 21,1 $\pm 1,8$.

В выборке лиц с дефицитом массы тела получены следующие результаты: по данным ультразвукового исследования среднее значение длины селезен-

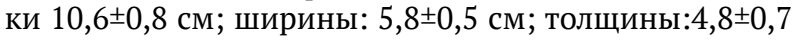
см; объема: $175,4 \pm 28,7$ см3. Для показателей антропометрии среднее значение роста - $167,7 \pm 6,9$ см; веса: $50,6 \pm 5,0$ кг. Медиана индекса массы тела составила $18,2 \pm 0,6$.

У выборки лиц с избыточной массой тела получены следующие результаты: в соответствии с данными ультразвукового исследования среднее значение длины органа равно 12,4 $\pm 0,7$ см; ширины: $6,8 \pm 0,8$ см;

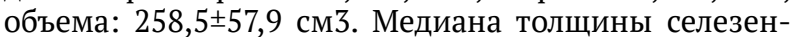
ки составила: $5,0 \pm 0,7$ см. По данным антропометрии среднее значение роста - 167,8 $\pm 5,3 \mathrm{~cm}$; веса: $76,0 \pm 5,0$ кг; индекса массы тела: 27,0 $\pm 1,4$.

Выводы. В результате исследования определены количественные параметры селезенки, ее возрастные особенности с учетом антропометрических данных у лиц женского пола первого периода зрелого возраста жителей Донецкого региона.

Ключевые слова: селезенка, ультразвуковое исследование, длина селезенки, ширина селезенки, толщина селезенки, объем селезенки, индекс массы тела.

\section{H.I. Kulish, O.O. Beshulia}

SEI HPE «M. Gorky Donetsk National Medical University», Donetsk

\section{QUANTITATIVE ANATOMY OF THE SPLEEN ACCORDING TO ULTRASOUND EXAMINATION OF FEMALE INDIVIDUALS OF THE DONETSK REGION}

The individual variability of the spleen's size and its derivatives, according to the body mass index of women of the 1st period of adulthood in the Donetsk region, haven't been practically studied, which makes the study of this issue relevant.

The aim of the study was to determine the quantitative parameters of the spleen according to the ultrasound method to establish the age norm taking into account the anthropometric parameters of women of the first period of adulthood age of the Donetsk region.

Material and methods. We used the survey, anthropometry measurement, ultrasound examination of the spleen and statistical analysis. Anthropometry includes height and weight measurement. Ultrasound examination was performed in the specific technique by a single operator in the B-gray scale mode in standard positions with longitudinal and transverse scanning. We measured length, width and thickness of the spleen. The obtained 
data was entered in Excel tables, and then s statistically processed in the program Statistica 10.0.

Results and discussion. We obtained the following results of individuals with normal body weight according to ultrasound data. The average spleen length is $10,8 \pm 1,0$ $\mathrm{cm}$; width: $5,7 \pm 0,5 \mathrm{~cm}$; thickness: $4,7 \pm 0,5 \mathrm{~cm}$; volume: $177,1 \pm 41,3 \mathrm{~cm} 3$. According to anthropometry: average height $-164,9 \pm 5,7 \mathrm{~cm}$; weight: $57,6 \pm 6,9 \mathrm{~kg}$; body mass index: $21,1 \pm 1,8$

Results of individuals with a body mass deficit weight according to ultrasound data. The average spleen length is $10,6 \pm 0,8 \mathrm{~cm}$; width: $5,8 \pm 0,5 \mathrm{~cm}$; thickness: $4,8 \pm 0,7$ $\mathrm{cm}$; volume: $175,4 \pm 28,7 \mathrm{~cm} 3$. For anthropometry indicators, the average height was $167,7 \pm 6,9 \mathrm{~cm}$; weight: $50,6 \pm$ $5,0 \mathrm{~kg}$. The Median body mass index was 18,2 $\pm 0,6$.
Results of individuals with overweight individuals to ultrasound data. The average spleen length is $12,4 \pm 0,7$ $\mathrm{cm}$; width: $6,8 \pm 0,8 \mathrm{~cm}$; volume: $258,5 \pm 57,9 \mathrm{~cm} 3$. The median spleen thickness was: $5,0 \pm 0,7 \mathrm{~cm}$. According to anthropometry, the average height was $167,8 \pm 5,3 \mathrm{~cm}$; weight: $76,0 \pm 5,0 \mathrm{~kg}$; body mass index: $27,0 \pm 1,4$.

Conclusions. As a result of the study the quantitative parameters of the spleen and its age characteristics were determined according to the anthropometric data of women of the first period of adulthood of the Donetsk region.

Key words: spleen, ultrasonography, spleen length, splenic width, thickness of the spleen, the volume of the spleen, the body mass index.

\section{ЛИТЕРАТУРА}

1. Mebius R.E., Kraal G. Structure and function of the spleen. Nature Reviews Immunology. 2005; 5: 606-616.

2. Смирнова Т.С., Ягмуров О.Д. Строение и функции селезенки. Морфология. 1993; 104 (5-6): 142-156.

3. Возгомент О. В., Пыков М. И., Зайцева Н.В., Акатова А.А., Ивашова Ю.А., Чигвинцев В.М. Новый ультразвуковой критерий оценки размеров селезенки у детей и определение диапазона нормативных значений органа. Педиатрическая фармакология. 2014; 11 (3): 89-92.

4. Камалов Ю.Р., Сандриков В.А. Руководство по абдоминальной ультразвуковой диагностике при заболеваниях печени. М.: Миклош; 2008.176.

5. Митьков В.В. Клиническое руководство по ультразвуковой диагностике. М.: Видар; 1996. 147.

6. Трофимов Т.Н. Лучевая анатомия человека. СПб.: Издательский дом СПб МАПО; 2005. 496.

7. Пальмер П.Е.С. Руководство по ультразвуковой диагностике. Женева; 2000. 456.

8. Биссет Р.А.Л., Хан А. Н. Дифференциальный диагноз при абдоминальном ультразвуковом исследовании. Витебск: Белмедктга; 1997. 272.

9. Митьков В.В. Практическое руководство по ультразвуковой диагностике. Общая ультразвуковая диагностика. М.: Видар; 2011. 720.

10. Наумович Е.Г. Комплексная ультразвуковая диагностика очаговых и диффузных заболеваний селезенки: дис. .... канд. мед. наук. М.; 2004. 117.

11. Radhika D. Vijayanirmala B. Morfometry of spleen. J. Evid. Based Med. Health; 2016; 3 (28): 1297-1300.

12. Sateesha N. B., Somayaji S. N., Soumya K. V. A study on the variations of size, shape and external features of the spleen in South Indian population. Int. J. Morphol; 2011. 29. 675-677.

13. Гунас И. В., Прокопенко С. В., Антонец Е. В., Дмитренко С. В. Связи сонографических параметров селезенки с конституциональными параметрами тела практически здоровых мужчин разных соматотипов. Мир медицины и биологии. 2017; 2(60).

14. Бунак В.В. Антропометрия. М.: Учмедгиз; 1941. 367.

15. Мартиросов Э.Г., Николаев Д.В., Руднев С.Г. Технологии и методы определения состава тела человека. М.: Наука; 2006. 248.

16. National Institutes of Health (NIH), National Heart, Lung, and Blood Institute (NHLBI). The practical guide: identification, evaluation, and treatment of overweight and obesity in adults. Bethesda: National Institutes of Health. 2000, NIH publication 00-4084.

17. Дергачев А. И., Котляров П. М. Справочник. Абдоминальная эхография. М: Эликс Ком; 2003. 350.

\section{REFERENCES}

1. Mebius R.E., Kraal G. Structure and function of the spleen. Nature Reviews Immunology. 2005; 5: 606-616.

2. Smirnova T.S., Yagmurov O.D. Stroenie i funktsii selezenki [Structure and function of the spleen]. Morfologiya. 1993; 104 (5-6): 142-156 (in Russian).

3. Vozgoment O. V., Pykov M. I., Zaitseva N.V., Akatova A.A., Ivashova Yu.A., Chigvintsev V.M.. Novyi ul'trazvukovoi kriterii otsenki razmerov selezenki u detei i opredelenie diapazona normativnykh znachenii organa [A new ultrasonic criterion of evaluating spleen dimensions in children and determination of the range of normal organ's dimensions]. Pediatricheskaya farmakologiya. 2014; 11 (3). 89-92 (in Russian).

4. Kamalov Yu.R., Sandrikov V.A. Rukovodstvo po abdominal'noi ul'trazvukovoi diagnostike pri zabolevaniyakh pecheni [Guidelines for abdominal ultrasound diagnostics for liver diseases]. Moskow: Miklosh; 2008.176 (in Russian).

5. Mit'kov V.V. Klinicheskoe rukovodstvo po ul'trazvukovoi diagnostike [Clinical manual of ultrasound diagnostics]. Moskow: Vidar; 1996. 147 (in Russian).

6. Trofimov T.N. Luchevaya anatomiya cheloveka [Human radiation anatomy]. SPb.: Izdatel'skii dom St.Petersburg: MAPO; 2005. 496 (in Russian).

7. Pal'mer P.E.S. Rukovodstvo po ul'trazvukovoi diagnostike [Manual of diagnostic ultrasound]. Zheneva; 2000. 456 (in Russian).

8. Bisset R.A.L., Khan A. N. Differentsial'nyi diagnoz pri abdominal'nom ul'trazvukovom issledovanii [Differential Diagnosis in Abdominal Ultrasound]. Vitebsk: Belmedktga; 1997. 272 (in Russian).

9. Mit'kov V.V. Prakticheskoe rukovodstvo po ul'trazvukovoi diagnostike. Obshchaya ul'trazvukovaya diagnostika [Practice manual of diagnostic ultrasound. General ultrasound diagnostics]. Moskow: Vidar; 2011. 720 (in Russian).

10. Naumovich E.G. Kompleksnaya ul'trazvukovaya diagnostika ochagovykh i diffuznykh zabolevanii selezenki: dis.... kand. med. nauk [Complex Ultrasound Diagnosis of Focal and Difuse Diseases of the Spleen]. Moskow; 2004. 117 (in Russian).

11. Radhika D. Vijayanirmala B. Morfometry of spleen. J. Evid. Based Med. Health; 2016; 3 (28): 1297-1300.

12. Sateesha N.B., Somayaji S.N., Soumya K.V. A study on the variations of size, shape and external features of the spleen in South Indian population. Int. J. Morphol; 2011. 29. 675-677.

13. Gunas I.V., Prokopenko S.V., Antonets E.V., Dmitrenko S. V. Svyazi sonograficheskikh parametrov selezenki s konstitutsional'nymi parametrami tela prakticheski zdorovykh muzhchin raznykh somatotipov [Relations spleen sonographic parameters with constitutional parameters of a body of practically healthy men of different somatotypes]. Mir meditsiny i biologii. 2017. 2(60) (in Russian). 
14. Bunak V.V. Antropometriya [Anthropometry]. Moskow: Uchmedgiz; 1941. 367 (in Russian).

15. Martirosov E.G., Nikolaev D.V., Rudnev S.G. Tekhnologii i metody opredeleniya sostava tela cheloveka [Technologies and methods of human body compositions]. Moskow: Nauka; 2006. 248 (in Russian).

16. National Institutes of Health (NIH), National Heart, Lung, and Blood Institute (NHLBI). The practical guide: identification, evaluation, and treatment of overweight and obesity in adults. Bethesda: National Institutes of Health. 2000, NIH publication 00-4084.

17. Dergachev A. I., Kotlyarov P. M. Spravochnik. Abdominal'naya ekhografiya [Abdominal echography: a Handbook]. Moskow: Eliks Kom; 2003. 350 (in Russian). 